\title{
Chemical Precipitation Synthesis of Fe, Ni, and Co Doped Zinc Sulphide Nanoparticles
}

\author{
A.Pricilla Jeyakumari ${ }^{1}$, M.Sumathi ${ }^{1}$, N.Thirumagal ${ }^{1}$, M.Uthaya Kumar $^{1}$ \\ ${ }^{1}$ Department of Physics, Thiruvalluvar Government Arts College, Rasipuram, Tamil Nadu, India
}

\begin{abstract}
Transition metal ions like Nickel, Cobalt and rare earth ions doped ZnS semiconductor materials have a wide range of applications in electroluminescence devices. In the present investigation nanoparticles of $\mathrm{Fe}$, Ni, Co doped ZnShave been synthesized successfully by simple chemical precipitation method. Particle size and specific surface area have been calculated from XRD analyses which confirm the nano structure of the samples. Absorption spectra of $\mathrm{Fe}$, Ni and Co doped ZnS were recorded and their optical transmittance are properly determined. The optical transmittance properties of the ZnS nanomaterial improved the addition of transition metal ion doping. The optical transmittance lies in visible region is one of the advantages of display devices and visible light LED'S. The molecular structure of the title compound was determined by the FT-IR analysis and the different vibrational bands confirmed the functional groups present in the sample. Current voltage graph of Fe doped $\mathrm{ZnS}$ were also drawn specific capacitanceof the sample were calculated. It shows enhanced electrical property of the nanomaterial. The Photo Luminescent spectra were also recorded it showed that the material has good photoluminescence property suitable for preparing optoelectronic devices. The antibacterial activity of Fe doped $\mathrm{ZnS}$ were also determined.
\end{abstract}

Keywords-Anti bacterial Activity, FT-IR, Nanoparticle, PL, Semiconductor, XRD, Zinc Sulphide

\section{Introduction}

In recent times there have been extensive studies on luminescent semiconductor nanocrystals because of their potential application in future optoelectronic devices. The nanosized semiconductor crystallites could change optical properties which are different from bulk materials[1]. Transition metal ions $(\mathrm{Ni}, \mathrm{CO})$ and rare earth ions doped $\mathrm{ZnS}$ semiconductor materials have a wide range of application in electroluminescence devices, phosphors, light emitting displays and optical sensors. Semiconductor nanoparticles doped with transition metal ions have attracted wide attention due to their luminescence properties[2]. Various efforts have been made by the researchers to dope transition metal ions in nanomaterial, several researchers have reported the luminescence quenching in $\mathrm{ZnS}$ nanoparticles due to $\mathrm{Fe}$ and $\mathrm{Ni}$ doping. Generally, $\mathrm{ZnS}$ becomes good host material, since it is a kind of wide band gap II-IV component semiconductor materials $(\mathrm{Eg}-3.6 \mathrm{eV})$, and with its energy band characteristics. It is commercially used as phosphor and thin film electroluminescence devices[3].Motivated by this, $\mathrm{Fe}, \mathrm{Ni}$ and $\mathrm{Co}$ doped $\mathrm{ZnS}$ nanoparticles were synthesized by Co-precipitation method their structural and morphological studies have been analyzed by powder XRD and SEM. Their optical properties were carried by UV-vis and photoluminescence spectra. The antibacterial activity of Fe doped ZnS were studied and their Zone of inhibition were also determined. The electrochemical property of the sample helped to estimate the specific capacitance value of the nanomaterials.

\section{(i) Synthesis of Fe doped $\mathrm{ZnS}$}

\section{Experimental}

Samples of different sized ZnS nanoparticles were prepared by simple chemical precipitation method using analytical grade Zinc Chloride, Ferric Chloride and Sodium Sulfide. 0.1M Fe doped ZnS nanoparticles were prepared by mixing $0.1 \mathrm{M}$ of $\mathrm{ZnCl}_{2}(20 \mathrm{ml})$ and $0.1 \mathrm{M}$ of $\mathrm{FeCl}_{3}(20 \mathrm{ml})$. Then, the obtained solution was continuously stirred for 1 hour, after that $90^{\circ} \mathrm{c}$ heat was supplied, along with that $0.1 \mathrm{M}$ of $\mathrm{NaS}(10 \mathrm{ml})$ was slowly added to the solution and the resultant solution was continuously stirred for another 1 hour. Finally, Orange color precipitate was obtained [4]. The obtained precipitate was allowed to evaporate at room temperature to obtain $\mathrm{Fe}$ doped $\mathrm{ZnS}$ nanoparticles in orange color powder form. The same procedure was adopted to get $0.01 \mathrm{M}$ sample. But the nanoparticle shows a brown color. Change in molarity causes some change in optical property. The same procedure is applied for the doped nanomaterials preparation of $0.01 \mathrm{M}$, $0.1 \mathrm{M}, 0.3 \mathrm{M}, 1 \mathrm{M} \mathrm{Fe}$ doped $\mathrm{ZnS}$. Instead of Fe various dopants like $\mathrm{Ni}$ and Co were added to get a green and purple color nanopowder of $\mathrm{ZnS}$ respectively.

National Conference on Current Advancements in Physics $3^{\text {rd }} \& 4^{\text {th }}$ February 2017 64 | Page Department of Physics, St. John's College, Palayamkottai-627 002, Tamilnadu, India. DOI 10.9790/4861-17002016470 


\subsection{Powder X-Raydiffraction (XRD) Pattern}

\section{Structural Characterizations}

The XRD Pattern of sample. $0.1 \mathrm{M} \mathrm{FeCl} 3$ doped $\mathrm{ZnS}$ is shown in figure 3.1. As expected, the XRD peaks of nanoparticles are considerably broadened due to finite size of these particles[5]. They were also compared with JCPDS file No.01-0792.

XRD- Particle Size Calculation

From this study, considering the peak at degrees, particle size has been estimated by using Debye Scherrer formula and found to be in the range of $21-24 \mathrm{~nm}$.

$$
\mathrm{D}=0.9 \lambda / \beta \cos \theta
$$

Were ' $\lambda$ ' is wave length of X-Ray $(0.1541 \mathrm{~nm})$, ' $\beta$ ' is FWHM (full width at half maximum), ' $\theta$ ' is the diffraction angle and ' $\mathrm{D}$ ' is particle diameter size. The value of $d$ (inter-planner spacing between the atoms) is calculated using Bragg's Law[6].

$$
2 \mathrm{~d} \sin \theta=\mathrm{n} \lambda
$$

Due to size effect the peaks broaden and then widths become larger as the particle size becomes smaller. The broadening of the peak may also occur due to micro strains of the crystal structure arising from defects like dislocation and twinning.. The XRD shows that $\mathrm{ZnS}$ nanoparticle has Cubic Phase[6].

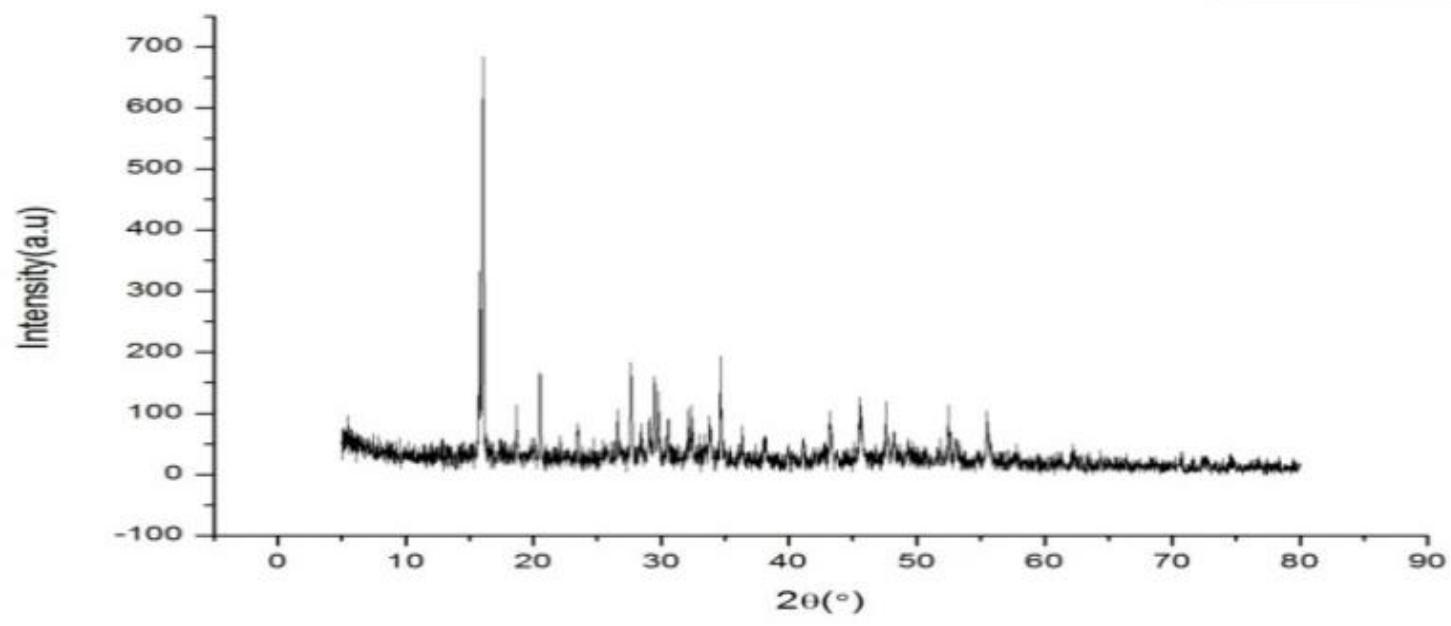

\subsection{FTIR spectral analysis}

Fig 3.1 Powder XRD Pattern of Fedoped ZnS

FTIR spectra of synthesized compound were recorded the range 400-4000nm employing a JASCO-FTIR 460 plus spectrometer. Following the $\mathrm{KBr}$ pellet technique at $300 \mathrm{k}$ with the scanning speed $2 \mathrm{~mm} / \mathrm{s}$ as shown in fig ( 3.2 ).

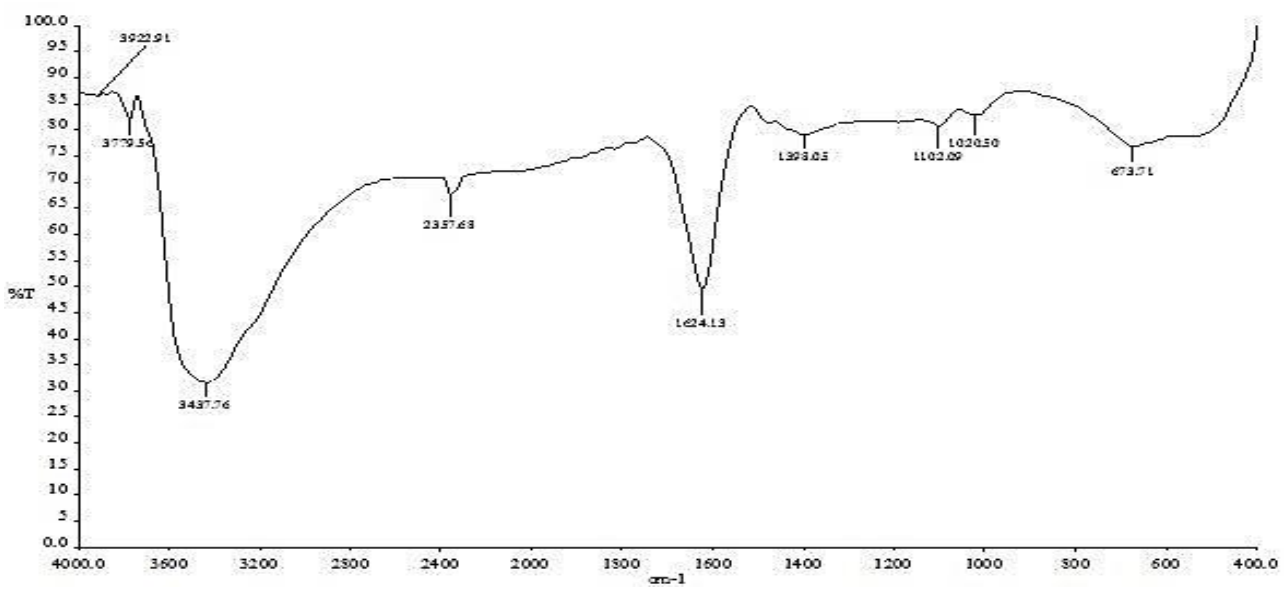

Fig 3.2 FTIR Spectrum of Fe doped ZnS 


\subsection{UV- Vis spectroscopy}

\section{Optical Properties}

UV-Vis spectrum of $\mathrm{Fe}, \mathrm{Ni}$ and Co doped ZnS Optical transmittance and absorption spectra of the Fe, Niand Co doped ZnSwere recorded using a Lambda $35 \mathrm{UV}-\mathrm{Vis}$ NIR spectrophotometer in the UV-Vis region.The UV-Vis absorption spectra of $\mathrm{Fe}, \mathrm{Ni}$, Co doped $\mathrm{ZnS}$ shows the absorption band at 300nm,220nm and $380 \mathrm{~nm}$, respectively and after that it shows less absorption, I.e. (highly transparent). In the transmittance spectra of Fe doped $\mathrm{ZnS}$ shown in fig (4.2(a)), and Ni doped $\mathrm{ZnS}$ shown in fig (4.2 (b)) and Co dopedZnS shown in fig (4.2 (c)) closely agree with the absorption spectra. It has a large optical transmittance window in the region $380-1100 \mathrm{~nm}$. There is no remarkable absorption band found in between. Wide transparency region is the important criteria of nanomaterial. Hence, it can be used in optical nano device fabrications. The sample is completely transparent in the visible region, which is mandatory for using in the electrochemical sensors and high sensitivity optical sensors for the detection[8]. The optical energy band gap of the given[9] Fe, Ni and Co doped $\mathrm{ZnS}$ of the nano materials is as shown Fig(4.3 a, b, c ) and tabulated in the table 4.2.

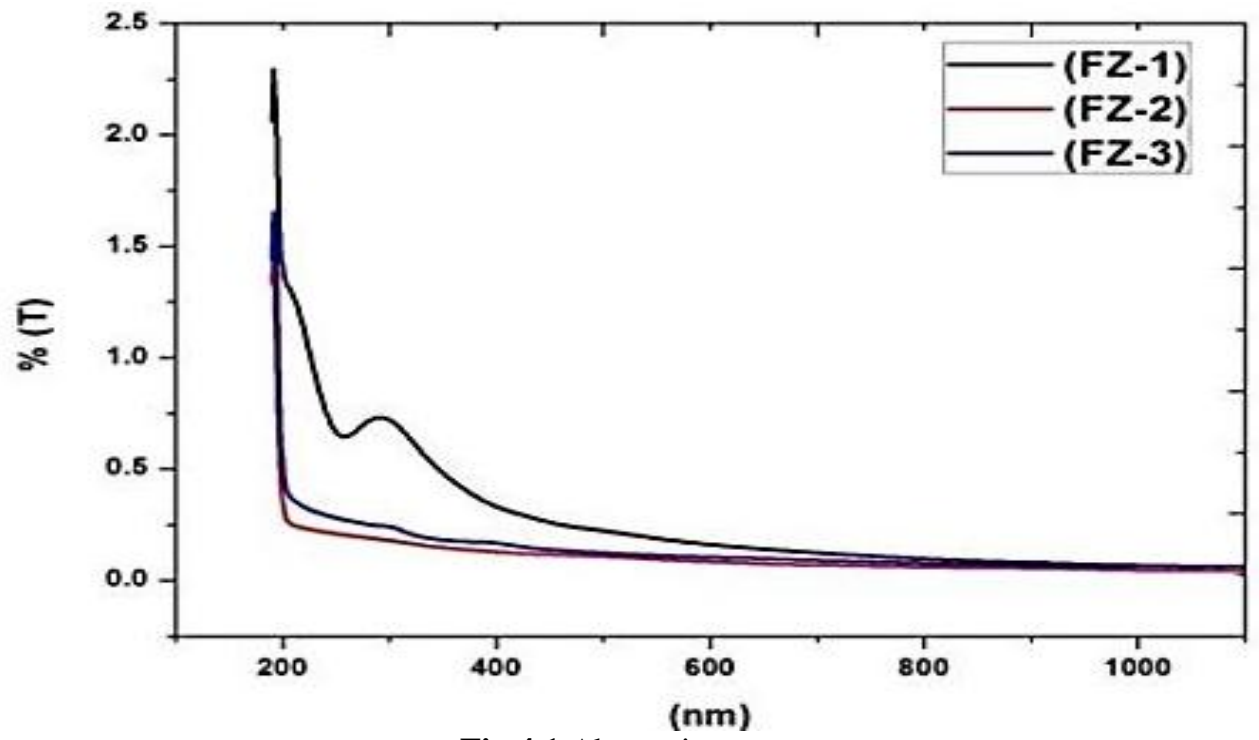

Fig.4.1 Absorption spectra

Table 4.1

\begin{tabular}{|l|l|l|}
\hline Nano particles & Optical transmitance window(nm) & Energy gap(ev) \\
\hline $\mathrm{FeCl}_{3}$ doped $\mathrm{ZnS}$ & $300-1200$ & 4.7 \\
\hline $\mathrm{NiCl}_{4}$ doped $\mathrm{ZnS}$ & $360-1200$ & 6.1 \\
\hline $\mathrm{CoCl}_{2}$ doped $\mathrm{ZnS}$ & $380-1200$ & 6.2 \\
\hline
\end{tabular}

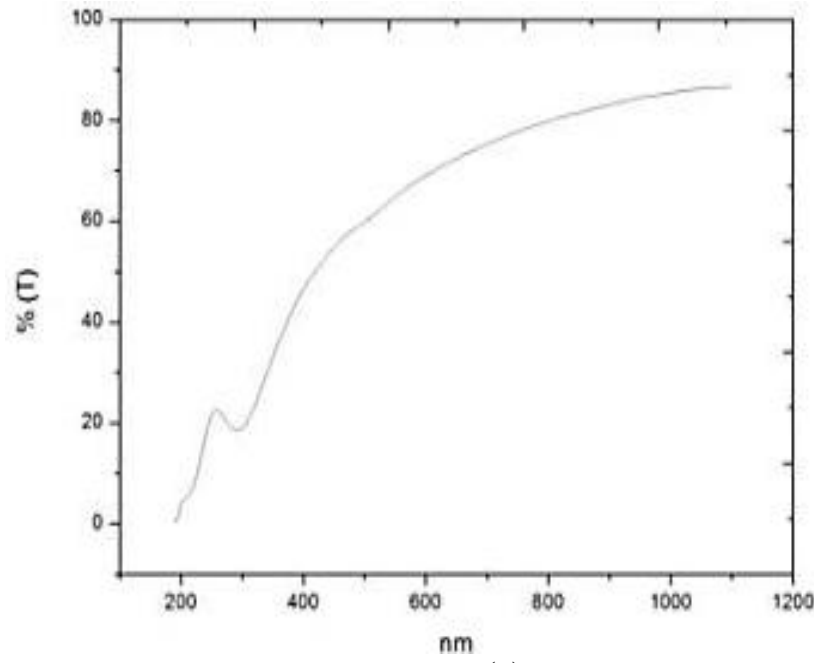

(a)

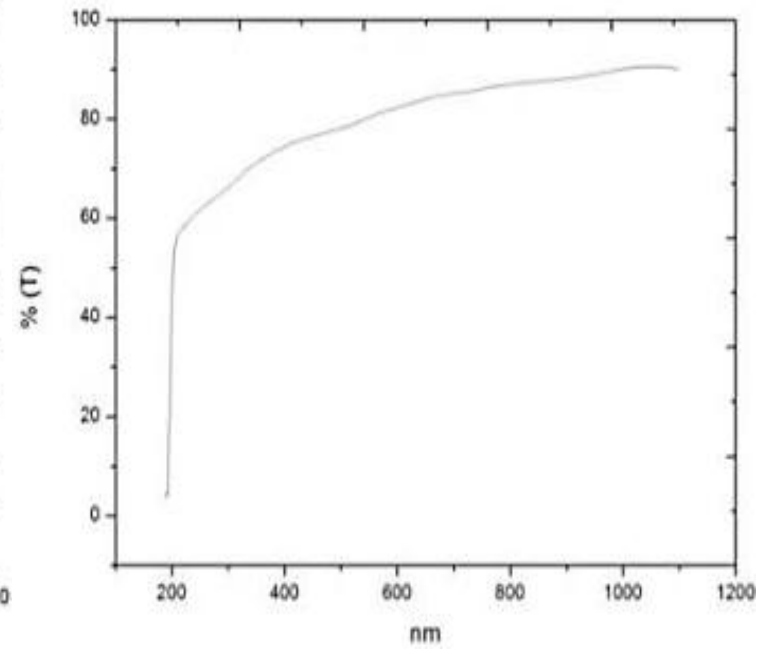

(b) 


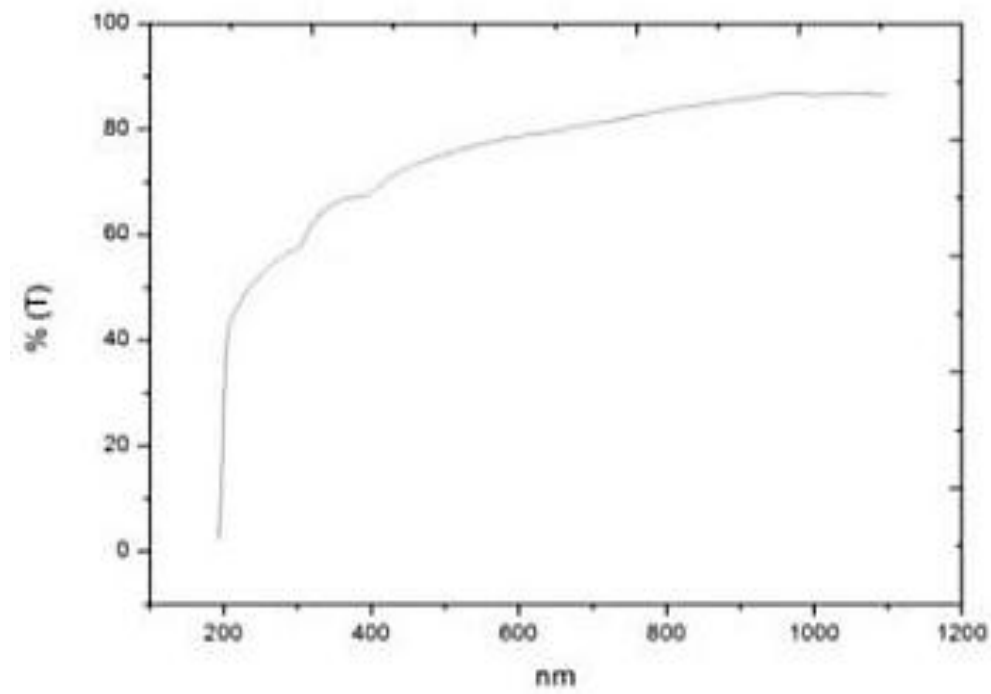

(c)

Fig. 4.2 Transmittance Spectra of (a) Fe-ZnS, (b) Ni-ZnS, (c) Co-ZnS respectively

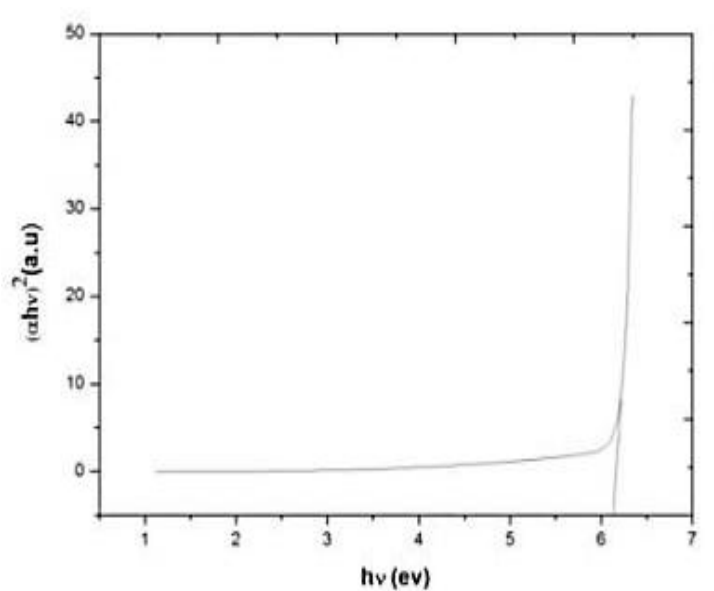

(a)

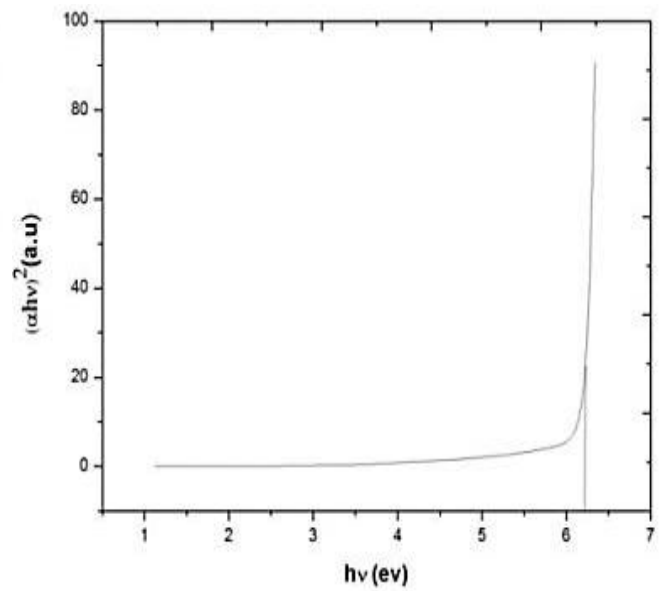

(b)

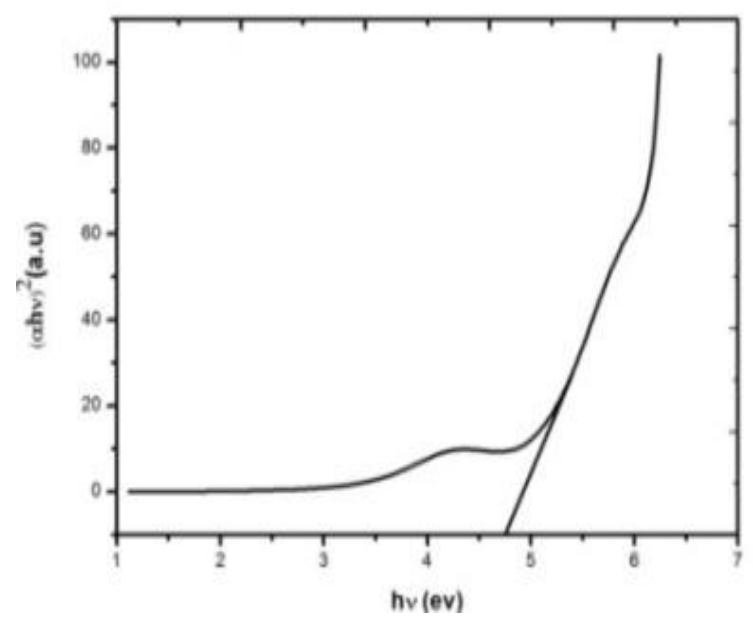

(c)

Fig.4.3 Energy band gap determination of (a) Ni-ZnS,(b) Co-ZnS, (c) Fe-ZnS respectively

\subsection{Photoluminescence Spectrum}


Photoluminescence spectrum of Fe, Ni, Co, doped ZnSare shown in Fig.(4.6) Some emission peaks are observed in photoluminescence spectrum.[10,11]A strong peak of $\mathrm{FeCl}_{3}$ doped $\mathrm{ZnS}(\mathrm{FZ}-1)$ at $426 \mathrm{~nm}$, near the band edge due to free excition emission is observed in photoluminescence spectrum.[16-19] The broad band in the region of 400-800 nm, in photoluminescence spectrum of $\mathrm{FeCl}_{3}$ doped $\mathrm{ZnS}$ is related to the amount of non-stochio-chiometric intrinsic defects and the same may be due to pure Fe doped $\mathrm{ZnS}$ as reported.[12] From photoluminescence analysis, it is observed that all are have fine luminescence property. Similarly all Fe doped $\mathrm{ZnS}$ are exhibiting the luminescence spectrum at $512 \mathrm{~nm}$ Niand $532 \mathrm{~nm}$ Co doped ZnS (FZ-3)respectively. Generally Ni doped ZnS(FZ-2)shows the ultraviolet emission with the red shift emission with compared to UV absorptions values in the photoluminescence analysis[13].

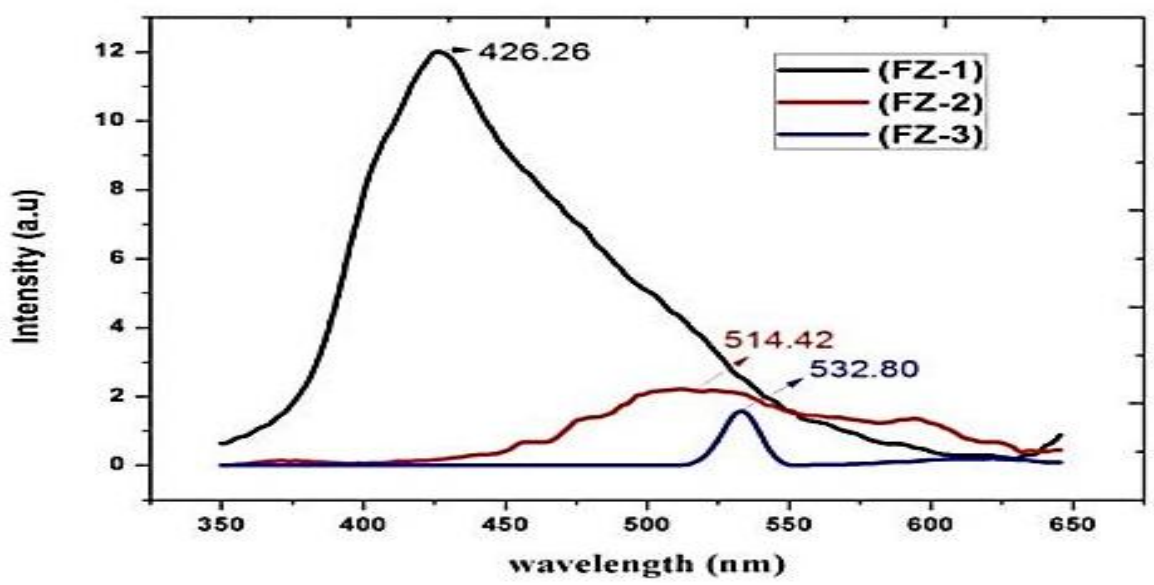

Fig., 4.6 Photoluminescence

\section{Procedure}

\section{Anti Bacterial Activity}

$1.3 \mathrm{~g}$ of Nutrient broth (Himedia) was mixed into $100 \mathrm{ml}$ of distilled water is kept for autoclave at $121^{\circ} \mathrm{c}$.[14] After autoclaving this was allowed to cool for bearable temperature then it was poured into sterile petriplates. The petripltes were allowed to solidification. After solidification, this plates were used for culture suaping for testing sensitive or resistant for given sample.[15,16]

Table 5.1

\begin{tabular}{|l|l|l|l|l|}
\hline Zone of inhibition in $\mathrm{mm}$ & \multicolumn{5}{|l|}{} \\
\hline \multirow{2}{*}{ Name of the microbes } & Different concentration & $\mathrm{mg}$ \\
\cline { 2 - 5 } & $0.5 \mathrm{mg}$ & $1 \mathrm{mg}$ & $2 \mathrm{mg}$ & $31 \pm 0.40$ \\
\hline Staphylococcus aureus $(\mathrm{Gram}+\mathrm{ve})$ & $17 \pm 0.02$ & $21 \pm 0.00$ & $28 \pm 0.20$ & $32 \pm 0.00$ \\
\hline Salmonella typhi(Gram -ve) & $18 \pm 0.40$ & $22 \pm 0.20$ & $26 \pm 0.35$ & $26 \pm 0.33$ \\
\hline Bacillus subtilis & $13 \pm 0.28$ & $20 \pm 0.16$ & $23 \pm 0.16$ & $28 \pm 0.00$ \\
\hline Escherichia coli & $18 \pm 0.00$ & $21 \pm 0.28$ & $24 \pm 0.33$ & \\
\hline
\end{tabular}

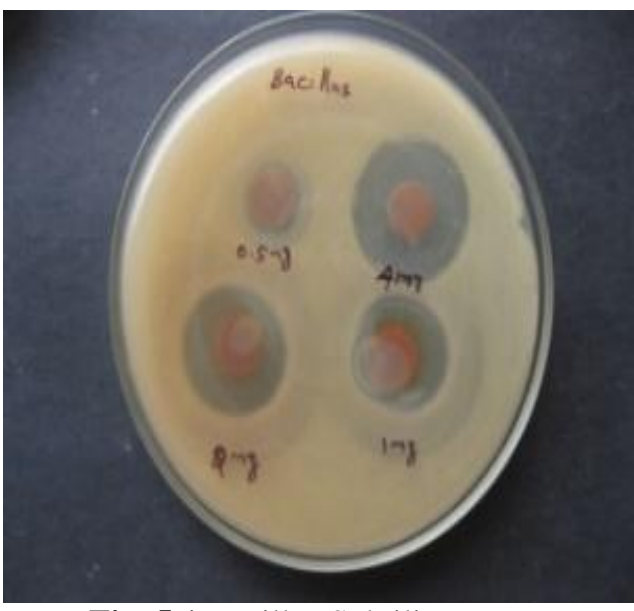

Fig, 5.1 Bacillus Subtilies

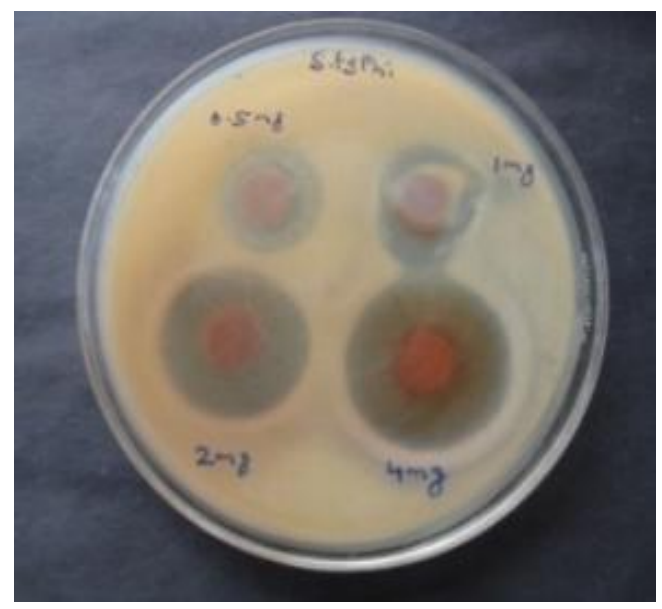

Fig. 5.2 Salmonella typhi 

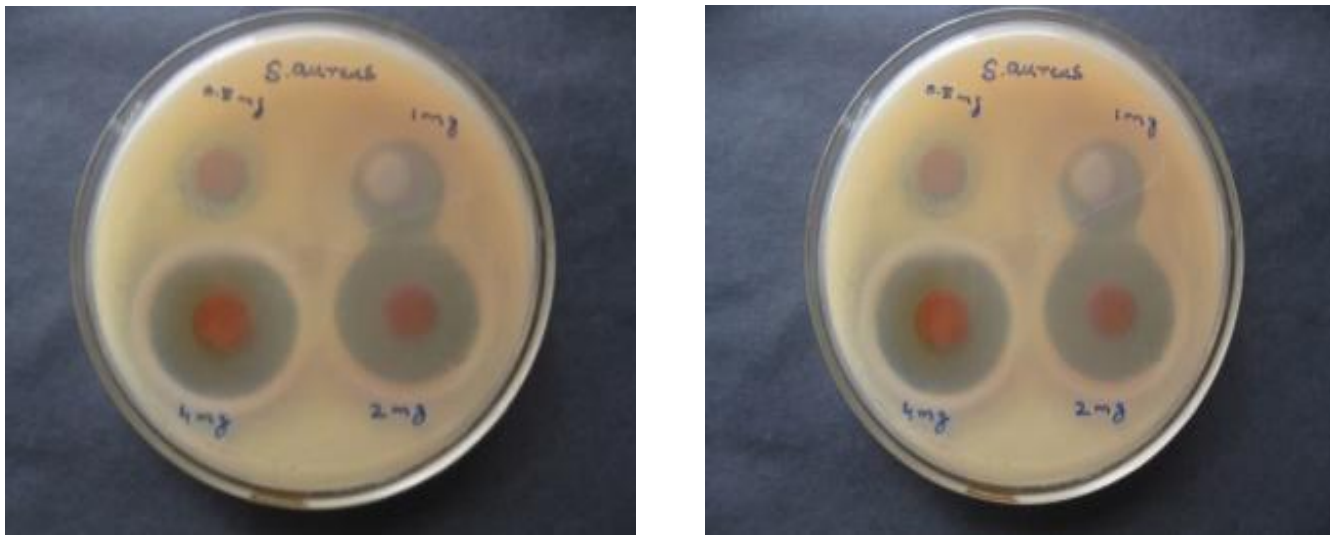

Fig, 5.3 Staphylococcus oureusFig. 5.4 Escherichia coli

\subsection{Electrochemecial Property-Cyclic Voltammetry}

The electrochemical properties of the Fe dopedZnS, Ni doped ZnS, Co doped ZnS nanomaterial as an electrode material for super capacitors were investigated using cyclic voltmeter (CV) and shown in the fig (5.1). It can be clearly understood that both the curves are rectangular in shape without any radix peak currents exhibiting the ideal capacitive behavior of the prepared electrodes. The integrated area under the CV curves is proportional to the specific capacitance. Fig (5.1) shows the CV curve of the above three electrodes under different scan rates in $1 \mathrm{M} \mathrm{Na} 2 \mathrm{SO} 4$ and all the curves are rectangular in shape and exhibit mirror image characteristics which indicate that the Faraday radix reactions re electrochemically reversible as well as an ideal electrochemical capacitive behavior. [17] It can be seen that the current under curve increases with as increase in the scan rate which indicates that the voltammetry current is always directly proportional to the scan rete and the CVs of the synthesized nanomaterial's retain a similar shape even at high scan rate, indicating an excellent capacitance behavior.

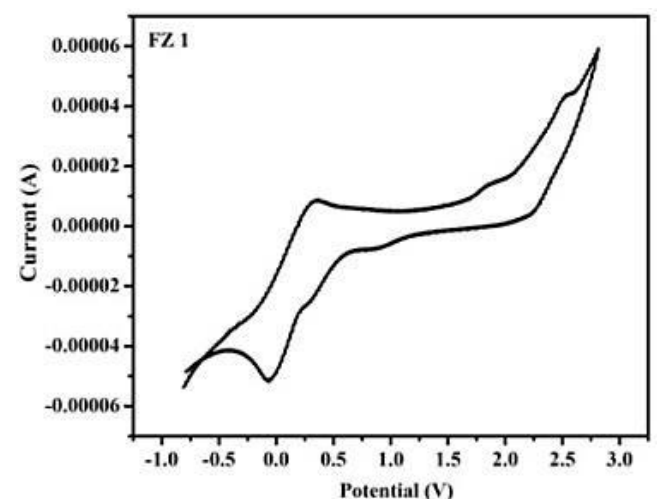

(a)

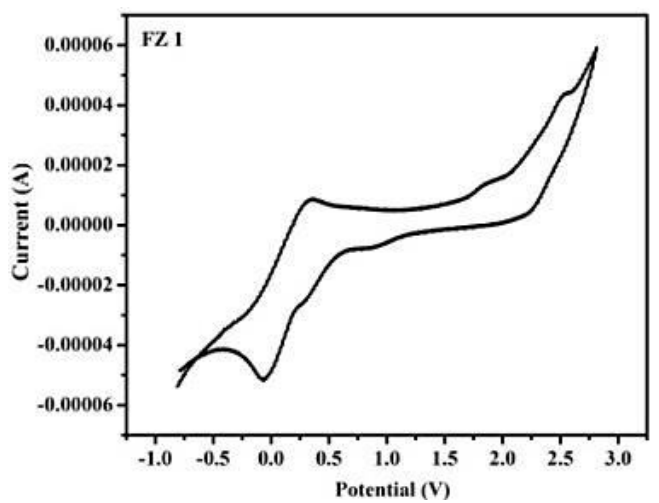

(b)

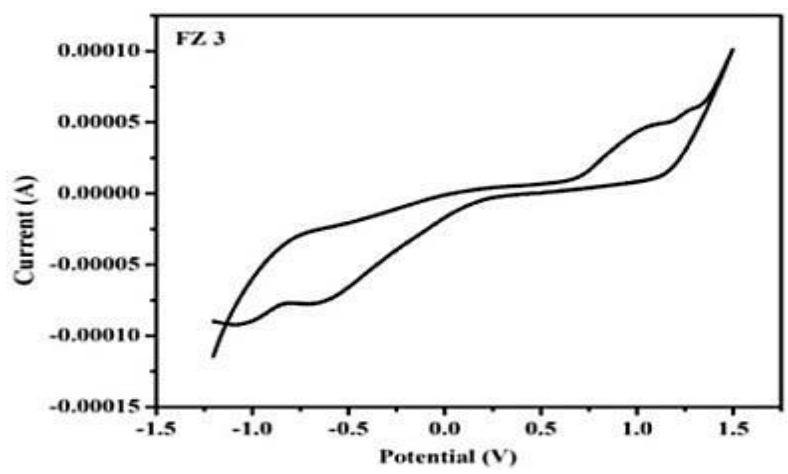

(c)

Fig 5.1 CV of(a).Fe doped ZnS (FZ-1) (b).Ni doped ZnS (FZ-2)(c).Co doped ZnS(FZ-3)respectively 


\section{Conclusion}

In the present investigation, Ferric( $\mathrm{Fe})$ doped Zinc sulphide $(\mathrm{ZnS})$ nanomaterial was synthesized by chemical precipitation metal at room temperature.Nanoparticles of $\mathrm{Fe}, \mathrm{Ni}$, Co doped have been synthesized successfully by simple chemical precipitation method. The powder X-ray diffraction pattern of the samples confirm, the formation of Nanoparticles of $\mathrm{FeCl}_{3}$ doped $\mathrm{ZnS}$ and their cubic structure. Particle size has been calculated from XRD analyses which confirm thenano structure of the samples. UV-Visible spectra of $\mathrm{Fe}, \mathrm{Ni}$ and $\mathrm{Co}$ doped $\mathrm{ZnS}$ were recorded and their optical transmittance are properly were determined Results show that $\mathrm{Ni}$ doped $\mathrm{ZnS}$ nanomaterial have a good optical transmittance property compare to Fe and Co dopedZnS. Therefore the optical transmittance of the nanomaterial improved the addition of translation metal iron doping. Consequently shifts the material transparency limits towards a blue wave length. Hence this material is also suitable for short wavelength generation. The optical transmittancelies in visible region is one of the advantages of display devices and visible light LED'S.

The molecular structure of the title compound was determined by the FT-IR analysis and the different vibrational bands confirmed the functional groups present in the sample.

Current voltage graph of Fe doped $\mathrm{ZnS}$ were also drawn the specific capacity of the sample were also calculated. It shows enhanced electrical property of the nanomaterial. The Photo Luminescent spectra were also recorded for the given materials. This nanomaterial has good photoluminescence property suitable for preparing optoelectronic devices.

Fe doped ZnS nanomaterial has better antibacterial activity. in the Staphylococcus oureus and Salmonellaetyphi microbes, Hence the synthesized Fe doped $\mathrm{ZnS}$ nanomaterial is suitable for preparing nanomedicine in order to improve the hemoglobin content of an anemic patient.

\section{References}

[1] Nano Technology for the life science (10 volume set), Chella S.S.R Kumar (editor) (Wily-VCH-2013).

[2] Warad H.C, Ghosh S.C, Hemtanon B, Thanachayanont C and DuttaJ,Sei.Technol.Ady. Mater., 2005, $296,6$.

[3] Nanda J, SapraS, Sarma D D.Chandrasekharan N and Hodes G. Chem, mater. 2011, 12(4),10181024.DOI; $10,1021 / \mathrm{cm} 990583 \mathrm{f}$.

[4] Yu I, Senna M, Appl. Phys. Lett.1999, 424,66.

[5] Dhanam M. Davitha B, Neethajose and Devasia D P, Chalcogenide Letter, 2009, 713,6(12).

[6] Alagar M. Theivasanthi T and Kubera Raja A, J of App, Sci., 2012, 398,12.

[7] Theivasanthi T and Alagar M, Int.J. of the Phys Sci., 2011, 726,6(15).

[8] Theivasanthi T and Alagar M. arXiy, 2011, 1111.0260.

[9] Ghosh S C, Thanachayanont C and Dutta J, ECTI Annual Conference, Thailand (ECTI-CON 2004).

[10] Skoog D A, Holler F J and Nieman T A, Priniciples of Instrumental Analysis, HarteourtBrade College Publishing, 2011.

[11] Theivasanthi T and Alagar M. Nano Biomed, Eng., 2011, 163,3(3).

[12] Antony J, Nutting J, Baer D R, Meyer D, Sharma A and Qiang Y, J. Nanomat., 2006, 2006(54961),1-4DOI; 10,1155/JNM/2006/54961.

[13] Park J Y, Lee Y J, Jun K W, Baeg J O and Yim D J,J, Ind, Eng. Chem., 2009,882,12.

[14] Liu A S and Oliveira M A S, J. Brazilian chem., Society, 207, 18(1), 143-152.

[15] Tsunekawa S, Wang J T and Kawazoe Y, J.Alloys and compounds, 2009, 408,11.

[16] Vogel, Quantitative Inorganic Analysis, Pearson Education, Delhi, 2010.

[17] Ramesh S and Wong K C, Ionics, 2012, 15(2), 249-254, DOI;10.1007/s11581-00-0268-2.

[18] hayatSarkar F.S.Z., Synthesis and magnetic Properties Investigations of Fe3o4 Nanoparticles, (2012).

[19] D.O.A.P.24. Cristina Blanco-Andujar, Elucidating the morphological and Structural evolution of nanoparticles .J. Mater. Chem, 2212498,(2012).

[20] C.C.B.A.S.G.Curtis, "Functionalisation of Magnetic Nanoparticles for Applications in Biomedicine," Journal of Physics D; Applied Physics, 36(13), 198-206, (2013).

[21] Ahamed M. 2011. Toxic response of nickel nanoparticles in human lung epithelial A549 cells. Toxicol in Vitro 25:930-936.

[22] Clift MJD, Byles MSP, Brown DM, Stone V. 2010. An investigation into the potential for different surface coated quantum dots to cause oxidative stress and affect macrophage cell signalling in vitro. Nanotoxicology 4(2):139-149.

[23] Cuenca AG, Jiang H, Hochwald SN, Delano M, Cance WG, Grobmyer SR. 2014. Emerging implications of nanotechnology on cancer diagnostics and therapeutics. Cancer 107:459-466.

[24] Hurley JF, Cherrie JW, Donaldson K, Seaton A, Tran CL. 2013. Assessment of health effects of long-term occupational exposure to tunnel dust in the London Underground. Institute of Occupational Medicine Research Report TM/03/02.

[25] Lead JR, Smith E. 2013. Environmental and Human Health Impacts of Nanotechnology (Chapter 1.8.1). Wiley, UK. Chapter 1. 